application to experiment. Chapter 19 on nuclear structure seems less successful. It contains a very useful up-to-date account of the achievements and physical concepts underlying the shell model and the collective model; but here the balance between mathematical formalism and physics, mentioned above, has not been achieved. Not enough mathematics is given for the reader to be able to appreciate how the qualitative concepts lead to far-reaching detailed quantitative predictions. The relevant theory is, of course, highly specialized and intricate, but just for that reason a bowdlerized version would have been most welcome. The authors have rightly refrained from an extensive application of meson physics to nuclear theory: as it is understated in the preface, "field theory is still no sure guide to correct results in nuclear physics". An attractive feature of this book is the use, wherever possible, of the effective range approximation, enabling the reader to become familiar with this very useful approach.

Nuclear physics covers a vast field, and our understanding of it has not yet reached a stage which allows a uniform presentation. This makes a first introduction to this subject difficult. It is most valuable to have a slender book, such as this second edition of "Elementary Nuclear Theory", which will serve this purpose admirably. It gives lucid and concise introductory accounts, the stress always being on the physical essentials, of most topics in nuclear theory which at the present time appear of fundamental importance.

F. MANDI

\section{SWANS, GEESE AND DUCKS}

\section{The Water Fowl of the World}

By Jean Delacour; illustrated by Peter Scott. Vol. 1 : The Magpie Goose, Whistling Ducks, Swans and Geese, Sheldgeese and Shelducks. Pp. $284+$ 16 plates. 105s. Vol. 2 : The Dabbling Ducks. Pp. $232+24$ plates. 1268 . net.

(London: Country Life, Ltd., 1954 and 1956.)

A THIRD volume will complete this monograph of a family of birds, the Anatidae, which makes a special appeal to the sportsman and the aviculturist as well as being of much ornithological interest. The work is handsomely produced, with text by one and illustrations in colour by another well-known authority. There is an account of every species and subspecies, giving its characteristics, distribution, general habits and record in captivity-this last a special feature, often with additional information on habits. Maps illustrate the distribution, but some of these have the defect of ignoring overlap between the breeding and non-breeding ranges.

The family is largely made up of a few familiar groups with numerous representatives widely spread. Thus, in the first volume, we find the whistling ducks of southern countries ; the swans, a small but cosmopolitan group; the true geese, grey and black; and the assemblage of species covered by the general title of sheldgeese and shelducks. Again, in the second volume, we have the dabbling or surface. feeding ducks, mostly placed in a single genus including such well-known species as mallard, teal, wigeon, pintail and shoveller, with related forms throughout the world.

There is, however, a notable addition of peculiar forms of limited range. Thus, the magpie goose of
Australasia is put in a sub-family by itself; the unique coscoroba of southern South America is apart from the typical swans; and monotypic genera are required for the Cape Barren goose, the blue-winged goose found only in Abyssinia, the Orinoco goose, the Egyptian goose and the South American crested duck. There is also the interesting genus of steamer ducks of southern South America and the Falkland Islands, once the subject of controversy but now considered to comprise three species-two flightless and one volant.

Other peculiar species, dealt with in the second volume, are the pink-headed duck of India, the pinkeared duck and freckled duck of Austrelia, the mountain blue duck of New Zealand, and the little torrent duck, inhabiting the swift streams of the Andes from the Caribbean to Tierra del Fuego and divisible into six sub-species.

Although the members of the family have many obvious points in common, there is considerable diversity in habit and still more in appearance. Mr. Scott figures the different plumages of each form, and the plates of the young in down are a pleasing feature.

LANDSBOROUGH THOMSON

\section{THE LEAF}

\section{The Growth of Leaves}

Proceedings of the Third Easter School in Agricultural Science, University of Nottingham, 1956. Edited by Prof. F. L. Milthorpe. Pp. $x+223$. (London : Butterworths Scientific Publications; New York: Academic Press, Inc., 1956.) $40 s$.

7 HE organizers of this symposium on the growth of leaves were prompted by the consideration that in attempts to improve agricultural production too much attention has been concentrated on the photosynthetic processes, changes in which, under normal agricultural conditions, are relatively unimportant in determining yield when compared with the effects of variations in the extent of the surface of the photosynthetic organs. The symposium was arranged to emphasize this principle, and follows the development of leaves from their inception, through expansion, to maturity.

The first part of the work deals with general aspects of growth and differentiation. In the introductory chapter by F. G. Gregory there is an interesting discussion of the general concept of the leaf and of the principal characteristics of leaf growth. E. Bünning contributes a valuable summary of his views on differentiation, while J. K. Heyes and R. Brown discuss the growth and differentiation of cells, using data obtained from their studies on roots. The second part of the symposium is concerned with the initiation of leaf primordia and opens with a chapter by C. W. Wardlaw on the inception of leaf primordia. in relation to metabolic and geometrical aspects of apical organization. This is followed by a mathematical treatment by $F$. J. Richards of the spatial correlations involved in the production of leaf pattern at the apex. N. Sunderland, J. K. Heyes and R. Brown conclude the section with an account of their original observations on growth and metabolism in the shoot apex of Lupinus. In the third section of the symposium, which deals with leaf expansion, $H$. Jones has covered a very wide field within the limits of his chapter on morphological aspects of leaf 